68th International Astronautical Congress (IAC), Adelaide, Australia, 25-29 September 2017. Copyright (C) 2017 by the authors. Published by the IAF, with permission and released to the IAF to publish in all forms.

IAC-17-A3,4B, $5, \mathrm{x} 39772$

\title{
DEVELOPMENT AND TESTING OF A PYRO-DRIVEN LAUNCHER FOR HARPOON-BASED COMET SAMPLE ACQUISITION
}

\author{
Stefan Völk*1, Stephan Ulamec ${ }^{2}$, Jens Biele ${ }^{3}$, Matthias Hecht ${ }^{4}$, Peter Lell ${ }^{5}$, Josef \\ Fleischmann ${ }^{6}$, Sebastian Althapp ${ }^{7}$, Markus Grebenstein ${ }^{8}$, Joseph A. Nuth ${ }^{9}$, Donald C. \\ Wegel $^{10}$, Walter F. Smith ${ }^{11}$, Lloyd R. Purves ${ }^{12}$, Douglas S. Adams ${ }^{13}$, Stuart Hill ${ }^{14}$, James C. \\ Leary $^{15}$, Harold A. Weaver ${ }^{16}$, and Scott A. Sandford ${ }^{17}$ \\ ${ }^{1}$ German Aerospace Center (DLR), Space Operations and Astronaut Training, Germany, stefan.voelk@dlr.de \\ ${ }^{2}$ DLR, Space Operations and Astronaut Training, Germany, stephan.ulamec@dlr.de \\ ${ }^{3}$ DLR, Space Operations and Astronaut Training, Germany, jens.biele@dlr.de \\ ${ }^{4}$ DLR, Space Operations and Astronaut Training, Germany, matthias.hecht@dlr.de \\ ${ }^{5}$ PyroGlobe GmbH, Hettenshausen, Germany, p.lell@gmx.de \\ ${ }^{6}$ Panasonic Industrial Devices Europe GmbH Pfaffenhofen, Germany, josef.fleischmann@eu.panasonic.com \\ ${ }^{7}$ Technische Universität München (TUM), Germany, s.althapp@tum.de \\ ${ }^{8}$ DLR, Robotics and Mechatronics, Germany, markus.grebenstein@dlr.de \\ ${ }^{9}$ NASA Goddard Space Flight Center (NASA GSFC), USA, joseph.a.nuth@nasa.gov \\ ${ }^{10}$ NASA Goddard Space Flight Center (NASA GSFC), USA, donald.c.wegel@nasa.gov \\ ${ }^{11}$ NASA Goddard Space Flight Center (NASA GSFC), USA, walter.f.smith@nasa.gov \\ ${ }^{12}$ NASA Goddard Space Flight Center (NASA GSFC), USA, lloyd.r.purves@nasa.gov \\ ${ }^{13}$ The Johns Hopkins University Applied Physics Laboratory (APL), USA, doug.adams@jhuapl.edu \\ ${ }^{14}$ The Johns Hopkins University Applied Physics Laboratory (APL), USA, stuart.hill@jhuapl.edu \\ ${ }^{15}$ The Johns Hopkins University Applied Physics Laboratory (APL), USA, james.leary@jhuapl.edu \\ ${ }^{16}$ The Johns Hopkins University Applied Physics Laboratory (APL), USA, hal.weaver@jhuapl.edu \\ ${ }^{17}$ NASA Ames Research Center (NASA ARC), USA, scott.a.sandford@nasa.gov \\ * Corresponding Author
}

The CORSAIR (COmet Rendezvous, Sample Acquisition, Investigation, and Return) mission is a proposal for the fourth NASA New Frontiers program. It belongs to the Comet Surface Sample Return mission theme which focuses on acquiring and returning to Earth a macroscopic sample from the surface of a comet nucleus. CORSAIR uses a harpoon-based Sample Acquisition System (SAS) with the spacecraft hovering several meters above the comet surface. This stand-off strategy overcomes disadvantages of systems using drills or shovels. Since comets are low gravity objects, these techniques would require anchoring before sampling, which is not necessary here. Moreover, the harpoon-based system allows for acquiring several samples from different locations on the comet maximizing the scientific output of the mission.

Each SAS assembly consists of a pyro-driven launcher, a Sample Acquisition and Retrieval Projectile (SARP) and a retraction system using a deployable composite boom structure. In order to collect enough cometary material, the launcher has to provide the required kinetic energy to the SARP. Due to high energy densities, pyrotechnically actuated devices ultimately reduce the overall system mass and dimensions. Here, an overview of the development, design and testing of the launcher is given. Furthermore, the launcher theory is introduced explaining the entire reaction chain: initiation $\rightarrow$ gas dynamics $\rightarrow$ SARP motion.

\section{INTRODUCTION}

Comets are invaluable time capsules that preserve materials from the dawn of the Solar System [1]. CORSAIR's proposed mission is straightforward: to return to Earth for analysis these early Solar
System relics from a comet nucleus [2, 3]. If selected, CORSAIR would return the first macroscopic comet samples directly from the nucleus of comet $88 \mathrm{P} /$ Howell, as well as coma dust samples. Volatile ices would be sublimated from the samples and chemically characterized before return. 88P/Howell 
68th International Astronautical Congress (IAC), Adelaide, Australia, 25-29 September 2017. Copyright (C) 2017 by the authors. Published by the IAF, with permission and released to the IAF to publish in all forms.

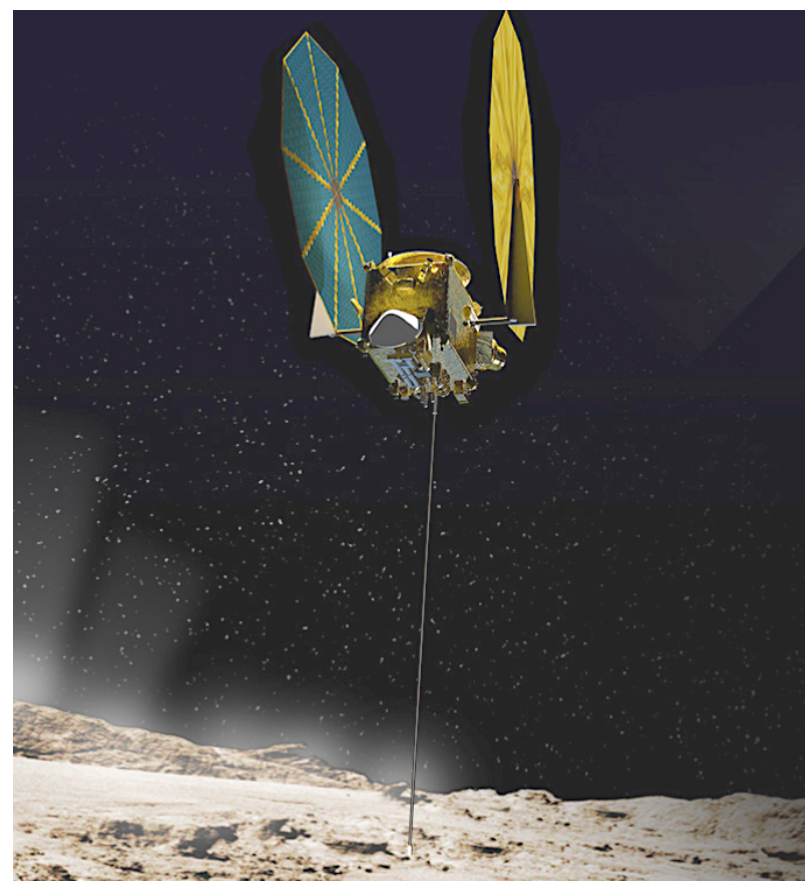

Fig. 1: Artist impression of CORSAIR sampling event.

is ideal for CORSAIR's proposed mission because it is a highly accessible, regularly observed, active Jupiter-family comet that will provide new discoveries from this first exploration.

CORSAIR would reveal the composition and organic inventory of comets through state-of-the-art analyses of the returned samples. Samples from two distinct surface locations would be returned to investigate variability across the nucleus. Each nucleus sample would contain $\geq 45 \mathrm{~g}$ of material to enable numerous analytical measurements using specialized terrestrial equipment that cannot be easily miniaturized and are far more accurate than their spaceborne counterparts, while also curating $\geq 75 \%$ of the sample material for future scientific investigations.

The proposed mission timeline includes launch of the mission on 19 July 2024, rendezvous at comet 88P/Howell from 30 May 2031 to 19 March 2032, and Earth return on 3 July 2036. During rendezvous and monitoring, CORSAIR's payload would map the comet's shape, activity, global morphology, colors, thermal properties, topography, and any changes to these properties over the mission. Coma gas composition and dust flux measurements would be made con-

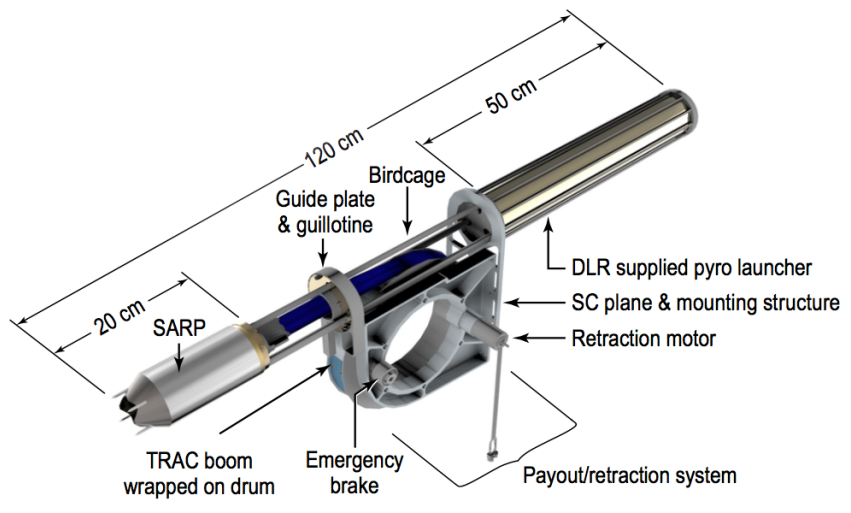

Fig. 2: SAS assembly with the subsystems SARP, retraction system (BRAD) and launcher.

tinuously during all phases of the comet rendezvous. During four flybys at different sites, images and laser altimetry would produce local, high-resolution topography maps of potential sampling sites. Subsequently, rehearsals of the descents would precede a sampling event. Images would be acquired before, during, and after each sampling event, to document the sampling process and its effect on the comet's surface. CORSAIR proposes to acquire two nucleus samples from surfaces with diverse properties.

\section{SAmple ACQUisition System}

CORSAIR's proposed Sample Acquisition System (SAS) is the culmination of years of studies, hardware development and testing. Sampling would be safely conducted without landing, while the spacecraft remained about $10 \mathrm{~m}$ above the surface. The SAS is designed to collect material down to depths of at least $10 \mathrm{~cm}$ to access more primitive material that may be below the altered surface $[2,3]$. The system is designed to sample over an extensive range of surface strengths and local topographies, from loose regolith to solid material. Four SAS assemblies each consisting of a pyro-driven launcher, a composite Boom Retraction And Deployment (BRAD) system, and a Sample Acquisition and Retrieval Projectile (SARP) are planned to be installed on the spacecraft. Figure 2 shows one entire assembly. Each SAS is a self-contained and independently operated unit.

The BRAD is shown in the middle part of figure 2 and consists of a Triangular Rollable And Collapsible (TRAC) boom [4] wrapped around a $30 \mathrm{~cm}$ diameter drum. The TRAC boom is chosen for flexibility during deployment and stiffness during retraction, nec- 
68th International Astronautical Congress (IAC), Adelaide, Australia, 25-29 September 2017. Copyright (C) 2017 by the authors. Published by the IAF, with permission and released to the IAF to publish in all forms.

essary to maintain control of the SARP throughout sampling. This reduces risk compared to any rigid coupling to the surface and prevents the SARP from impacting the spacecraft at any time. The SARP is the SAS projectile portion that collects the nucleus sample. The SARP outer sheath serves as the surface impactor and does not return to the spacecraft. Inside the outer sheath are mounted the inner sheath, cartridge, and mechanisms necessary to complete sampling. Each sample cartridge has a $298 \mathrm{~cm}^{3}$ sample bay and a spring-loaded knife-edge door at its opening that closes to encapsulate the captured sample. Both systems, BRAD and SARP are developed and tested at NASA GSFC. The SAS launcher is a DLR contribution to the proposed CORSAIR mission.

\section{SAMPLE ACQUISITION AND HANDLING}

The sampling event starts with a spacecraft command to the SAS at about $10 \mathrm{~m}$ above the comet surface. This triggers the launcher accelerating the SARP to penetrate $\geq 10 \mathrm{~cm}$ into the surface. The SARP outer sheath bears the impact forces and breaks up comet material for ingestion. An artist impression of the sampling event with deployed TRAC boom and SARP is shown in figure 1. The SARP comes to rest through comet resistance, retraction system braking, or both. A timer closes the cartridge door, cutting through the cometary material and fully encapsulating the sample. Subsequently, the inner part of the SARP separates from the outer sheath and the BRAD is activated for SARP retraction.

After the sample has been acquired, the spacecraft departs the comet and at a safe distance, imaging of the SARP is used to provide an initial assessment. These images are analyzed on the ground and, once reviewed, a robotic arm extracts the cartridge and transfers it to a Sampling Handling Station (SHS) for devolatilization and later from the SHS to the Sample Storage System (SSS) [5]. The robotic arm is a DLR contribution to the proposed mission. It grasps, removes, transfers, inserts and releases the cartridge fully autonomously through the use of force feedback and torque control without visual targeting [6-8].

\section{LAUNCHER DESIGN}

The launcher is part of the SAS and transfers the required momentum to the SARP and BRAD. A sufficiently high SARP velocity must be chosen, in order to penetrate the comet surface deep enough for material with the hardest expected compressive strength. The majority of estimates of the compressive strengths of cometary surface materials are less than tens of kilopascals [9-13]. However, Rosetta showed that the sublimation and redeposition cycles near the surface can cause sintering of icy material [14], resulting in compressive strengths of $\sim 1 \mathrm{MPa}$ or more $[15,16]$. The first touchdown of the Rosetta lander Philae on the surface of comet $67 \mathrm{P} /$ Churyumov-Gerasimenko revealed a granular soft surface with a compressive strength of $\sim 1 \mathrm{kPa}$ at least $20 \mathrm{~cm}$ thick, possibly on top of a more rigid layer [17]. Higher-strength material is present in some regions [14], but this material is not representative of the bulk nucleus [11]. Thus, a sampling system that penetrates a range of material strengths, including material $>1 \mathrm{MPa}$, is required to maximize the likelihood of acquiring the most primitive and unaltered cometary material that resides below the processed surface layers. From an engineering standpoint, a maximum expected compressive strength of $2 \mathrm{MPa}$ is assumed. Obtaining primitive cometary material requires the collection of samples to a depth of at least $10 \mathrm{~cm}[2,3]$. SARP penetration tests into porous glass foam material with compressive strength of $2.4 \mathrm{MPa}$ showed a penetration depth of $14.6 \mathrm{~cm}$ at an impact velocity of $33.4 \mathrm{~m} / \mathrm{s}$. For the launcher, the design velocity is in the range between $35 \mathrm{~m} / \mathrm{s}$ and $50 \mathrm{~m} / \mathrm{s}$.

Further important constraints and requirements are the minimization of impulse and force exerted on the spacecraft, the reduction of system mass and the contamination control of the retrieved samples. Mainly because of high-energy densities, it was decided early in the study phase to use a pyrotechnical system. Prior to that, alternatives such as electromagnetic, cold gas and spring systems have been studied as well. However, these concepts require considerable more allocated mass and - in case of an electromagnetic design - would not be compatible with existing constraints concerning the spacecraft avionics.

\section{Design evolution}

Launcher development began at DLR in 2013. The initial SAS concept had a dual tether retraction system consisting of two metallic tapes laterally attached to the launcher structure. The tapes are rolled up in stowed position and deploy during sampling with the ends attached to the SARP. Because 
68th International Astronautical Congress (IAC), Adelaide, Australia, 25-29 September 2017. Copyright (C) 2017 by the authors. Published by the IAF, with permission and released to the IAF to publish in all forms.

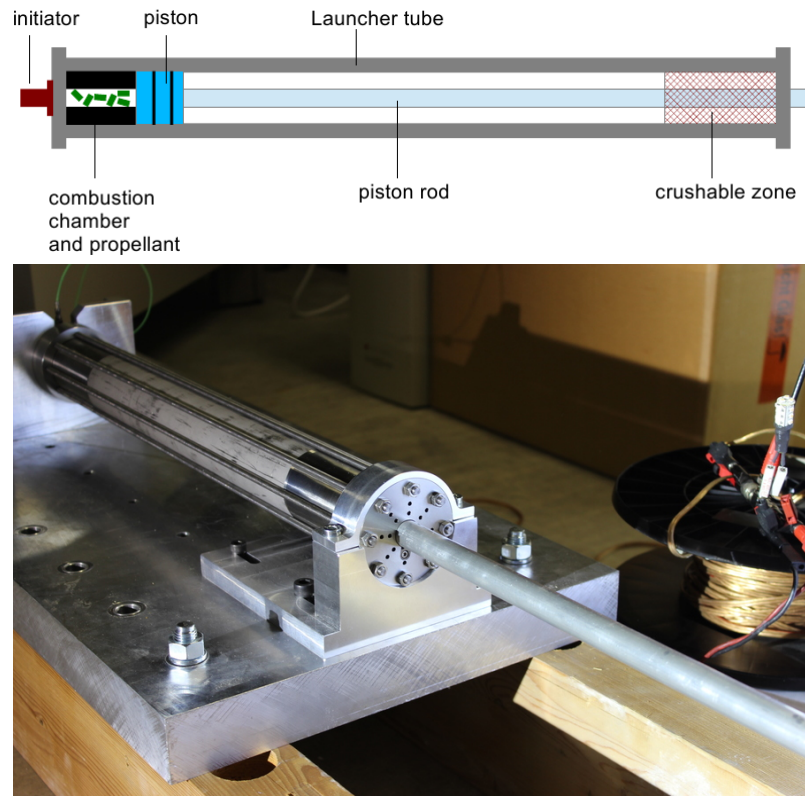

Fig. 3: Top: initial launcher concept. Bottom: launcher version 1.0 with deployed piston rod.

of this lateral configuration, a smaller diameter launcher with a single piston rod was designed. The upper sketch in figure 3 shows the building blocks of this initial launcher concept. In contrast to the Rosetta/Philae harpoons [18], the propellant is ignited via an initiator instead of a bridge wire. Here, the commercial initiator PyroGlobe GG821 is used. In a later stage, this can be replaced by a space-qualified counterpart, e.g. the NASA Standard Initiator (NSI), or alternatively, space-qualification of a commercial available initiator is provided.

Main ingredients of the propellant mixture are 7-perforation Nitrocellulose grains which are placed inside a dedicated combustion chamber (see upper sketch in figure 3). The combustion chamber is a cylindrically shaped inlay and is manufactured from polyoxymethylene (POM). The combustion gas generates pressure pushing the piston with the attached piston rod. The prototype should prove the feasibility of the concept and no space-qualified items have been used. For sealing, rubber O-rings are used. Not shown in figure 3 is a dummy mass emulating the inertia of SARP and retraction system. At the end of the launcher tube, a crushable zone absorbs the kinetic energy of piston and rod after release of the dummy mass. Here, a stack of several Aluminum honeycomb rings is used.
For the first prototype - version 1.0 - the piston rod was manufactured from an Aluminum tube. However, with increasing propellant charge, this turned out not to withstand critical buckling loads. Therefore, version 1.5 was built with a piston rod made from high-modulus carbon composite material. The outstanding stiffness-to-mass-ratio proved to sustain higher loads. Furthermore, the crushable was replaced by an Aluminum honeycomb structure with higher density yielding higher crush strengths and shorter stopping distances.

\section{Baseline design}

Later, the retraction system design was changed to a single boom concept, providing better performance on both boom deployment and retraction. This had major implications on the launcher design. To accommodate the centrally guided composite boom, first, a launcher tube diameter twice as large as in the previous version had to be chosen, and second, the rod configuration had to be changed. Due to the increased inner tube diameter of $100 \mathrm{~mm}$, a much higher gas production was needed. Therefore, it was decided to implement a high-low pressure system with two separate chambers $[19,20]$.

In the launcher version 2.0 shown in figure 4 , the propellant burns in a combustion chamber, and the produced gas passes an injection head - a flow-restricting device - before entering the launcher tube. Because of the higher and steady gas pressure in the combustion chamber, propellant burning is optimized, whereas the pressure profile inside the launcher tube is more uniform, attenuating the acceleration peak. Ultimately, the high-low pressure approach reduces the mass of the launcher subsystem and solves the packing of the propellant, which is stored in a dedicated powder chamber. Pyrotechnic high-low pressure systems have been successfully operated in various NASA space missions, in particular for parachute mortar systems [21, 22].

Although launcher version 2.0 uses a different chamber design compared to former versions, some parts have been adopted from version 1.5, notably the high-modulus carbon composite rods and the higher strength crushable material. Launcher version 2.0 is composed of mass optimized and primarily of space-proof parts. Most parts are manufactured from Titanium grade 5. Thin walls down to $2 \mathrm{~mm}$ have been manufactured for the launcher tube. The piston includes three spring-energized and reinforced 
68th International Astronautical Congress (IAC), Adelaide, Australia, 25-29 September 2017. Copyright (C) 2017 by the authors. Published by the IAF, with permission and released to the IAF to publish in all forms.

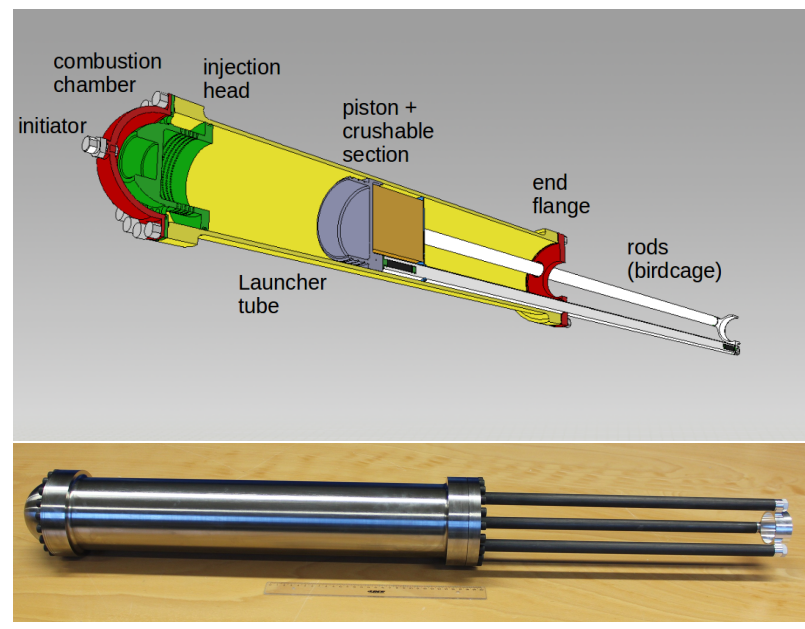

Fig. 4: Top: sectional view of launcher baseline design. Bottom: launcher version 2.0 hardware.

polytetrafluoroethylene (PTFE) seals. The momentum is transferred from the piston to the SARP via three piston rods arranged circularly around the centrically/axially guided boom. The boom is deployed from a sidewise mounted reel (see figure 2). For the boom feed-in, enough clearance in both the lateral and axial direction has to be provided in this rod configuration, which is also referred to as birdcage.

In order to provide enough axial offset, longer rods than for the first-generation launcher are required. Since longer rods increase buckling loads, the material selection and dimensioning of the rods have been carried out with special care. Figure 5 shows the numerical and analytical studies performed on this topic. The upper image is an example of an Finite Element Method (FEM) simulation with a lateral force applied to one end of the birdcage. Lateral forces are mostly relevant for the terrestrial test setup with horizontal launcher orientation and $1 \mathrm{~g}$ environment.

The lower plot in figure 5 shows the results of an analytical calculation using the Euler-buckling formula modified to composite materials $[23,24]$. The colored region denotes the allowed rod configurations as a function of inner and outer rod diameter, i.e. those dimensions fitting to the geometrical constraints of the BRAD system and withstanding buckling. Since the birdcage mass depends on the rod dimensions, the resulting kinetic energy and required length of the crushable are also functions of the rod
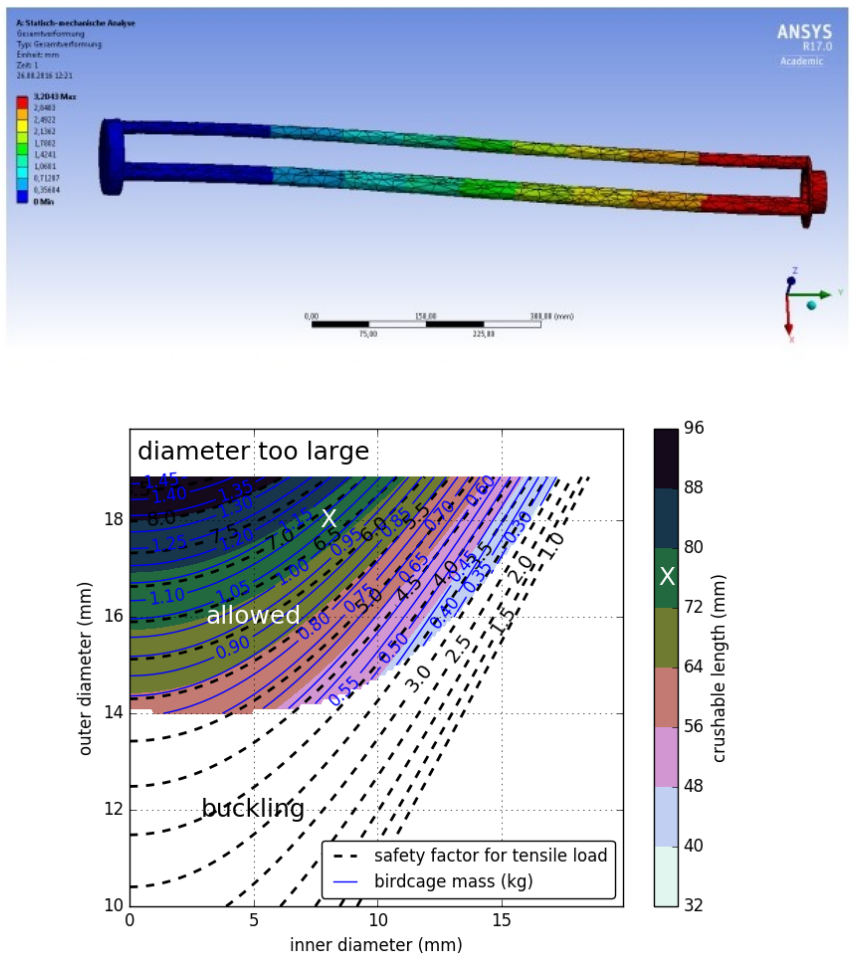

Fig. 5: Top: FEM simulation of mechanical loads on birdcage assembly. Bottom: dimensioning of carbon composite birdcage rods.

dimensions. This is reflected by the color code. In addition, the birdcage mass and the safety-factor for tensile load during deceleration are plotted on the same graph. The white $X$ sign marks the chosen rod configuration for the launcher hardware.

\section{LAUNCHER TESTING}

All launcher versions have been tested in standalone experiments, i.e., using dummy masses instead of the SARP and the retraction system. By doing this, SARP and launcher testing can be performed independently from each other. The rationale behind this approach is that the inertias of SARP and BRAD can be combined to an effective mass reflecting the mass of the SARP plus an extra mass, which is equivalent to the rotational inertia of the BRAD. Figure 6 shows the test setup with the horizontally mounted launcher. After completing the piston stroke, the dummy mass (elongated metallic body attached to the birdcage) is released. Then, the dummy mass is in free flight before it is absorbed in a box filled with padding material (not shown). 
68th International Astronautical Congress (IAC), Adelaide, Australia, 25-29 September 2017. Copyright (C) 2017 by the authors. Published by the IAF, with permission and released to the IAF to publish in all forms.

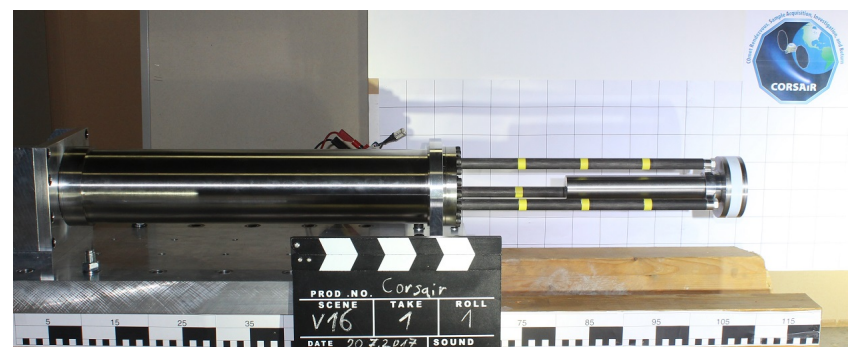

Fig. 6: Testing of launcher version 2.0

A set of experimental data is acquired at each test: A high-speed camera with frame rates up to 20000 fps records the motion of piston and dummy mass. Videos of tests performed with launcher versions 1.5 and 2.0 can be found in reference [25]. With the help of post-processing tracking, fitting and derivation algorithms, the velocity and acceleration profiles are deduced from the positional data. A piezoelectric pressure sensor monitors the pressure profile inside the combustion chamber. Pressure sensor and initiator are mounted on a T-piece converging to the combustion chamber. Optionally, the pyro-shock can be measured. This is basically a vibration spectrum of the launcher structure.

\section{LAUNCHER THEORY - INTERNAL BALLISTICS}

Traditionally, the design of pyrotechnic devices is a process driven to a large extent by trial and error. Experiments tend to be expensive, time-consuming and have high risk potential. Furthermore, some quantities such as time and space-resolved gas temperature are practically inaccessible or require complex and costly nonstandard instrumentation. In order to minimize these disadvantages and streamline the development process, numerical modeling of the internal ballistics became a main effort of this work. In the beginning, stand-alone program codes have been written for solving individual and elementary problems. When the project and the knowledge of underlying processes further developed, these efforts converged into a more complete and complex simulation framework. The overall goal of the modeling efforts is to reflect the entire reaction chain beginning with the burning of propellant grains to gas-dynamic processes and finally the piston and SARP motion. Main objectives are supporting the launcher development process and predicting the launcher behavior for future tests.

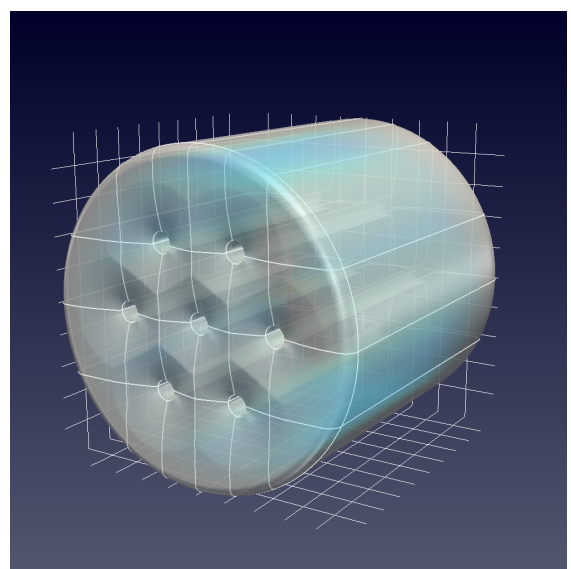

Fig. 7: Simulated burning of a 7-perforation propellant grain. The simulation results help to find the effective shape function of the propellant.

When facing the modeling task, the complexity of the program code typically increases in a non-linear fashion by every design iteration. This can be mitigated by using a declarative and object-oriented framework which is provided by the Modelica language. Modelica supports acausal modeling, i.e., solves implicit systems of equations. The acausality makes Modelica library classes more reusable than traditional classes containing assignment statements where the input-output causality is fixed [26, 27]. Furthermore, Modelica is non-proprietary and OpenModelica is open-source, which has been used here among other programming languages. The environment includes graphical tools for both programming and data visualization. There are detailed libraries for many domains such as electric circuits, mechanics, thermodynamics and fluids.

Here, an overview of implemented physical processes in the modeling framework is given. Solid propellant changes its geometric shape as the surface successively turns to gas (see figure 7). Thus, the total rate at which combustion gas is produced can be controlled by the geometric shape of the propellant grains. In general, the geometry is designed to achieve a progressive, degressive or neutral flow rate. Propellants come in complex geometric shapes and a mixture of different geometries with 7-perforation Nitrocellulose grains as main ingredient is used in the launcher. In order to reflect the change in geometry mathematically, an effective shape function $\phi(t)$ is used. Furthermore, the combustion rate depends on the chamber pressure. In interior ballis- 
68th International Astronautical Congress (IAC), Adelaide, Australia, 25-29 September 2017. Copyright (C) 2017 by the authors. Published by the IAF, with permission and released to the IAF to publish in all forms.

tics $[20,28,29]$, the regression rate usually follows the empirical formula as expressed in this correlation:

$$
\dot{r}=\beta p^{\alpha}
$$

with $\dot{r}$ being the regression rate of the propellant, $\beta$ the linear burn rate and $\alpha$ an exponent to the pressure $p$. The combustion gas is modeled as an ideal gas with fixed and averaged properties. Given the mole fractions of the molecular species, the equilibrium gas properties are either deduced from look-up tables or calculated by the NASA program code Chemical Equilibrium with Applications (CEA) [30]. It is assumed that there are no energy losses, for example through heat transfer or piston friction. Adiabatic conditions are primarily justified since the piston stroke is fast $(\sim 20 \mathrm{~ms})$ compared to heat exchange with the environment. Therefore, conservation of energy is assumed for the energy balances. Finally, Newton's law of motion correlates the force originating from the gas pressures with the acceleration of the piston.

In case of the baseline design, there is a flow restricting device which limits the volume flow rate. In the upper image of figure 4 , this device is referred to as injection head. 400 pinholes restrict the gas flow from the combustion chamber to the launcher tube / expansion chamber. The pinholes are modeled as an ideal adiabatic nozzle where a discharge coefficient $C_{\mathrm{D}}$ takes into account the shape of the holes [31]. Additionally, the pinholes are covered by a thin Aluminum foil which bursts on the individual holes at a particular pressure difference between both chambers. First, this mechanism prevents premature pressure compensation enhancing propellant burning in the combustion chamber. Second, the foil limits the amount of open pinholes favoring choked flow conditions.

\section{$\underline{\text { RESULTS }}$}

Table 1 summarizes key parameters and results of a consistent set of tests. Tests with changed propellant mixture or non-nominal piston rod behavior are not listed. An exception is test V9 with the highest dummy mass velocity reached. Since the observed failure of the piston rod occurred during piston stopping - i.e. after the acceleration phase the results of V9 are still consistent.

For each launcher version, propellant charge $m_{\mathrm{p}}$ was increased step by step. Furthermore, the combustion chamber volume $V_{\mathrm{CC}}$ of the first generation launcher can be adjusted by drilling the POM inlet (see upper sketch in figure 3). Before each test, the impact of propellant charge and chamber volume on the motion profiles have been analyzed using numerical models of the internal ballistics. By comparing tests V1 and V9, the relevance of the combustion chamber volume becomes clear. Both tests showed comparable values for peak acceleration $a_{\text {max }}$. However, test V9 had a $m_{\mathrm{p}} 3.67$ times higher and a $V_{\mathrm{CC}} 5.47$ times larger than in test V1. Having fixed launcher dimensions and dummy mass, a possible failure of the piston rod depends on $a_{\max }$ only. As a first approximation, the Euler-buckling formula can be applied here (compare also lower plot in figure 5). The combustion chamber volume is a relevant design parameter for the resulting peak acceleration.

For test V10, a high-modulus carbon composite rod was used instead of the metallic rod in launcher version 1.0. The piston assembly had the same mass as in the metallic version. Due to the increased stiffness, the rod withstands higher peak accelerations and due to the increased tensile strength of the assembly, a crush material with enhanced crush strength could be used resulting in shorter stopping distances and a longer free acceleration length $l_{\mathrm{f}}$. Figure 8 shows the motion profile of test V10. Solid lines correspond to experimental data. The upper plot shows the piston position which has been directly measured through the described tracking method. Both, piston velocity (middle plot) and acceleration (lower plot) are derived from the tracking data. From all tests performed with a one-chamber launcher (versions 1.0 and 1.5), V10 uses the most optimized configuration of parameters $m_{\mathrm{p}}$ and $V_{\mathrm{CC}}$. The final dummy mass velocity was $51 \mathrm{~m} / \mathrm{s}$, slightly exceeding the upper end of the design range, while the peak acceleration was comparably low. Dashed lines correspond to numerically modeled data. The curves do not exactly coincide. However, experiment and simulation are consistent, i.e., the profiles have comparable shapes and the absolute values are of comparable magnitude.

As listed in table 1, the pressure values in the two last tests with launcher version 2.0 are much lower than with the one-chamber design. The first values of $p_{\max }$ correspond to the measured combustion chamber pressures; the value in V11 is missing due to instrumentation reasons. The lower pressure as com- 
68th International Astronautical Congress (IAC), Adelaide, Australia, 25-29 September 2017. Copyright (C) 2017 by the authors. Published by the IAF, with permission and released to the IAF to publish in all forms.

Table 1: Selected launcher tests. First two columns denote test number and launcher version. Dummy mass was $2.987 \mathrm{~kg}$ for launcher versions $1.0-1.5$, and $4.65 \mathrm{~kg}$ for launcher version $2.0 . m_{\mathrm{p}}$ : propellant charge, $V_{\mathrm{CC}}$ : combustion chamber volume, $p_{\max }$ : peak pressure (two values for launcher version 2.0 corresponding to combustion and expansion chamber), $a_{\text {max }}$ : peak piston acceleration in multiples of gravity $(\mathrm{g}), l_{\mathrm{f}}$ : free acceleration length, $v_{\mathrm{DM}}$ : final dummy mass velocity.

\begin{tabular}{lcrrrrrl}
\hline test & ver. & $m_{\mathrm{p}}(\mathrm{g})$ & $V_{\mathrm{CC}}\left(\mathrm{cm}^{3}\right)$ & $p_{\max }(\mathrm{MPa})$ & $a_{\max }(\mathrm{g})$ & $l_{\mathrm{f}}(\mathrm{mm})$ & $v_{\mathrm{DM}}(\mathrm{m} / \mathrm{s})$ \\
\hline V1 & 1.0 & 3.0 & 8.1 & 15.5 & 771 & 376 & 35 \\
V3 & 1.0 & 6.5 & 22.6 & 11.0 & 573 & 376 & 45 \\
V9 & 1.0 & 11.0 & 44.3 & 15.0 & 777 & 376 & 62 \\
V10 & 1.5 & 8.3 & 44.3 & 11.0 & 540 & 448 & 51 \\
V11 & 2.0 & 5.7 & 222.0 & -1.1 & 142 & 406 & 20 \\
V16 & 2.0 & 15.0 & 222.0 & $5.3 / 2.8$ & 348 & 406 & 31.4 \\
\hline
\end{tabular}
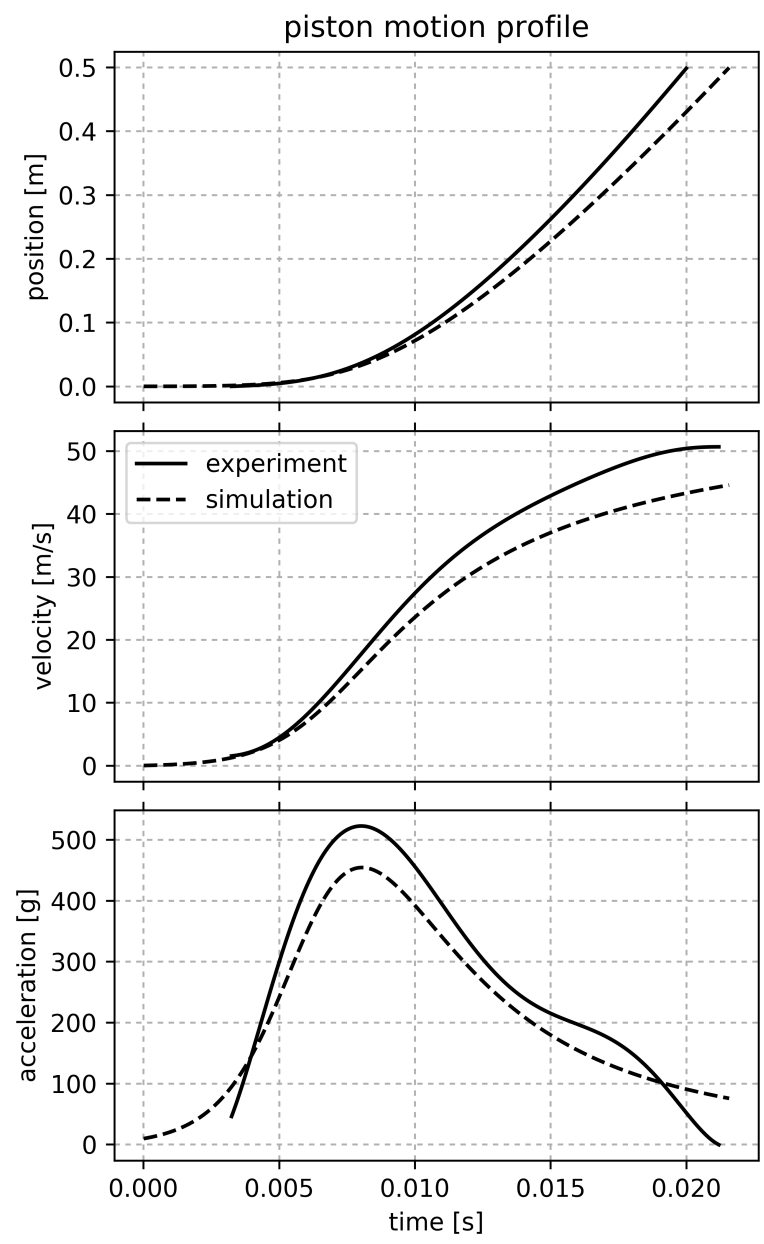

Fig. 8: Piston motion profile of test V10 (launcher version 1.5). Comparison of experimental with simulated data. pared to former launcher versions is the consequence of a larger combustion chamber volume amongst others. The second values are the peak pressures in the expansion chamber. These values have been reconstructed from the acceleration profile assuming a uniform pressure distribution within the expansion chamber. So far, the final dummy mass velocities have been lower than in tests V1 - V10. However, a much higher dummy mass of $4.65 \mathrm{~kg}$ has been used instead of the $2.987 \mathrm{~kg}$ mass taking into account the increased inertia of the modified retraction system. Furthermore, the mass of the piston assembly increased as well, and a higher gas production is needed in order to pressurize the larger volume.

\section{Conclusions AND OUtLOOK}

In summary, an overview of the proposed CORSAIR mission and the harpoon-based comet sampling method has been given. After describing the building blocks of the Sample Acquisition System, this paper highlights the pyro-driven launcher, which provides the required kinetic energy to the Sample Acquisition and Retrieval Projectile. Launcher design requirements are mainly derived from scientific requirements (sampling depth) and the maximum expected compressive strength of the comet surface. Subsequently, the design evolution of different launcher versions is presented, terminating in the current baseline design that is compatible with a novel retraction system using a single composite boom structure. Afterwards, the setup for launcher stand-alone tests is described and the modeling framework for the internal ballistics of the launcher is outlined. Finally, the test results are discussed and compared with simulation data. 
68th International Astronautical Congress (IAC), Adelaide, Australia, 25-29 September 2017. Copyright (C) 2017 by the authors. Published by the IAF, with permission and released to the IAF to publish in all forms.

In order to conform with the velocity design range, further tests with launcher version 2.0 are envisaged. By increasing the propellant charge, higher dummy mass / SARP velocities will be achieved. The complex correlations between propellant, launcher design parameters and piston motion must be analyzed carefully. The described modeling framework supports this process. It is planned to upgrade this framework for systematic scans and evaluation of the entire parameter space. The goal of this effort is to find a set of optimized design parameters.

\section{REFERENCES}

[1] S.A. Sandford, N.L. Chabot, N. Dello Russo, J.C. Leary, E.L. Reynolds, H.A. Weaver, D.H. Wooden, and The CORSAIR Team. Corsair (comet rendezvous, sample acquisition, investigation, and return): A new frontiers mission concept to collect samples from a comet and return them to earth for study. In 80th Annual Meeting of The Meteoritical Society, July 2017. LPI Contrib. No. 1987.

[2] G.H. Fountain and et al. Comet surface sample study report. Technical Report SDO-11998, NASA, 2008. prepared for NASAs Planetary Science Division, 30 April 2008, Washington, DC.

[3] J. Veverka, L. Johnson, and E. Reynolds. Comet surface sample return mission - nasa planetary science decadal survey mission concept study. Technical report, NASA, 2011. http://sites. nationalacademies.org/SSB/SSB_059331.

[4] J. Banik and T. Murphey. Performance validation of the triangular rollable and collapsible mast. 24th Annual AIAA/USU Conference on Small Satellites, 2010.

[5] D. Adams, J. Leary, S. Papadakis, C. Apland, R. Maddock, S. Kellas, R. Winski, T. White, C. Kazemba, and P. Agrawal. Comet surface sample return: Sample chain system overview. In Aerospace Conference, 2017 IEEE, pages 17. IEEE, 2017.

[6] G. Hirzinger, K. Landzettel, B. Brunner, M. Fischer, C. Preusche, D. Reintsema, A. AlbuSchäffer, G. Schreiber, and B.-M. Steinmetz. Dlr's robotics technologies for on-orbit servicing. Advanced Robotics, 18(2):139-174, 2004.

[7] K. Landzettel, A. Albu-Schäffer, B. Brunner, A. Beyer, R. Gruber, E. Krämer, C. Preusche,
D. Reintsema, J. Schott, H.J. Steinmetz, et al. Rokviss verification of advanced light weight robotic joints and tele-presence concepts for future space missions. In Proceedings of the 9th ESA Workshop on Advanced Space Technologies for Robotics and Automation (ASTRA), 2006.

[8] K. Landzettel, C. Preusche, A. Albu-Schaffer, D. Reintsema, B. Rebele, and G. Hirzinger. Robotic on-orbit servicing-dlr's experience and perspective. In Intelligent Robots and Systems, 2006 IEEE/RSJ International Conference on, pages 4587-4594. IEEE, 2006.

[9] M.F. A'Hearn, M.J.S. Belton, W.A. Delamere, J. Kissel, K.P. Klaasen, L.A. McFadden, K.J. Meech, H.J. Melosh, P.H. Schultz, J.M. Sunshine, et al. Deep impact: excavating comet tempel 1. science, 310(5746):258-264, 2005.

[10] J.E. Richardson and H.J. Melosh. An examination of the deep impact collision site on comet tempel 1 via stardust-next: Placing further constraints on cometary surface properties. Icarus, 222(2):492-501, 2013.

[11] O. Groussin, L. Jorda, A.-T. Auger, E. Kührt, R. Gaskell, C. Capanna, F. Scholten, F. Preusker, P. Lamy, S. Hviid, et al. Gravitational slopes, geomorphology, and material strengths of the nucleus of comet $67 \mathrm{p} /$ churyumov-gerasimenko from osiris observations. Astronomy 83 Astrophysics, 583:A32, 2015.

[12] E. Heggy, A. Shafie, A. Hérique, J. Lasue, W.W. Kofman, and A.C. Levasseur-Regourd. Constraining the bulk dust to ice ratio and compressive strength for comet churyumov gerasimenko using consert radar observations. In $A G U$ Fall Meeting Abstracts, 2015.

[13] R. Roll, L. Witte, and W. Arnold. Rosetta lander philae-soil strength analysis. Icarus, 280: 359-365, 2016.

[14] T. Spohn, J. Knollenberg, A.J. Ball, M. Banaszkiewicz, J. Benkhoff, M. Grott, J. Grygorczuk, C. Hüttig, A. Hagermann, G. Kargl, et al. Thermal and mechanical properties of the near-surface layers of comet $67 \mathrm{p} /$ churyumovgerasimenko. Science, 349(6247):aab0464, 2015.

[15] H.L. Jessberger and M. Kotthaus. Compressive strength of synthetic comet nucleus sam- 
68th International Astronautical Congress (IAC), Adelaide, Australia, 25-29 September 2017. Copyright (C) 2017 by the authors. Published by the IAF, with permission and released to the IAF to publish in all forms.

ples. Physics and Mechanics of Cometary Materials,(Hunt J. and Guyenne TD, eds.), pages 141-146, 1989.

[16] K. Seiferlin, T. Spohn, and J. Benkhoff. Cometary ice texture and the thermal evolution of comets. Advances in Space Research, 15(10): $35-38,1995$.

[17] J. Biele, S. Ulamec, M. Maibaum, R. Roll, L. Witte, E. Jurado, P. Muñoz, W. Arnold, H.U. Auster, C. Casas, et al. The landing (s) of philae and inferences about comet surface mechanical properties. Science, 349(6247):aaa9816, 2015 .

[18] M. Thiel, J. Stöcker, C. Rohe, N.I. Kömle, G. Kargl, O. Hillenmaier, and P. Lell. The rosetta lander anchoring system. In 10th European Space Mechanisms and Tribology Symposium, volume 524, pages 239-246, 2003.

[19] J. Corner. A theory of the internal ballistics of the "hoch-und-niederdruck kanone". Journal of the Franklin Institute, 246(3):233-248, 1948.

[20] G. Backstein et al. Handbook on weaponry. Rheinmetall GmbH, D, 1982.

[21] J.E. Pleasants. Parachute mortar design. Journal of Spacecraft and Rockets, 11(4):246-251, 1974.

[22] R.E. Vasas and J. Styner. Mars exploration rover parachute mortar deployer development. AIAA Paper, 2137:2003, 2003.

[23] B.W. Rosen. Mechanics of composite strengthening. In Fiber Comopsite Materials, chapter 3, pages 37-75. American Society of Metals, 1965.

[24] N.A. Fleck. Compresssive failure of fiber composites. In Advances in applied mechanics, volume 33, pages 43-117. New York, NY: Academic Press, 1997.

[25] J.A. Nuth. Video supplement: Gsfc development and testing of harpoon-based approaches for collecting comet samples, 2017. https://ssed.gsfc. nasa.gov/harpoon/Video_Supplement-V1.pdf.

[26] P. Fritzson and V. Engelson. Modelica - a unified object-oriented language for system modeling and simulation. In European Conference on Object-Oriented Programming, pages 67-90. Springer, 1998.
[27] P. Fritzson. Principles of object-oriented modeling and simulation with Modelica 3.3: a cyberphysical approach. John Wiley \& Sons, 2014.

[28] J. Corner. Theory of the interior ballistics of guns. John Wiley and Sons Inc., New York, 1950.

[29] D.E. Carlucci and S.S. Jacobson. Ballistics: theory and design of guns and ammunition. CRC Press, 2013.

[30] S. Gordon and B.J. MacBride. Computer program for calculation of complex chemical equilibrium compositions and applications. NASA Reference Publication 1 \& 2, NASA, October 1994.

[31] H. Dubbel. DUBBEL: Taschenbuch für den Maschinenbau. Springer-Verlag, 2013. 